\title{
A note on compactness properties of the singular Toda system
}

\author{
Luca Battaglia, Gabriele Mancini \\ S.I.S.S.A., Via Bonomea 265, 34136 Trieste (Italy) \\ e-mail:lbatta@sissa.it,gmancini@sissa.it
}

\begin{abstract}
In this note, we consider blow-up for solutions of the $S U(3)$ Toda system on a compact surface $\Sigma$. In particular, we give a complete proof of the compactness result stated by Jost, Lin and Wang in [11] and we extend it to the case of singularities. This is a necessary tool to find solutions through variational methods.
\end{abstract}

\section{Introduction}

Let $(\Sigma, g)$ be a smooth, compact Riemannian surface. We consider the $S U(3)$ Toda system on $\Sigma$ :

$$
-\Delta u_{i}=\sum_{j=1}^{2} a_{i j} \rho_{j}\left(\frac{V_{j} e^{u_{j}}}{\int_{\Sigma} V_{j} e^{u_{j}} d v_{g}}-\frac{1}{|\Sigma|}\right)-4 \pi \sum_{j=1}^{l} \alpha_{i j}\left(\delta_{p_{j}}-\frac{1}{|\Sigma|}\right) \quad i=1,2
$$

with $\rho_{i}>0,0<V_{i} \in C^{\infty}(\Sigma), \alpha_{i j}>-1, p_{j} \in \Sigma$ given and

$$
A=\left(a_{i j}\right)=\left(\begin{array}{cc}
2 & -1 \\
-1 & 2
\end{array}\right)
$$

is the $S U(3)$ Cartan matrix.

The Toda system is widely studied in both geometry (description of holomorphic curves in $\mathbb{C P}^{N}$, see e.g. [4, 6, 8]) and mathematical physics (non-abelian Chern-Simons vortices theory, see [10, 18, 19]).

In the regular case, Jost, Lin and Wang [11] proved the following important mass-quantization result for sequences of solutions of (1). 
Theorem 1.1. Suppose $\alpha_{i j}=0$ for any $i, j$ and let $u_{n}=\left(u_{1, n}, u_{2, n}\right)$ be a sequence of solutions of (1) with $\rho_{i}=\rho_{i, n}$. Define, for $x \in \Sigma$, $\sigma_{1}(x), \sigma_{2}(x)$ as

$$
\sigma_{i}(x):=\lim _{r \rightarrow 0} \lim _{n \rightarrow+\infty} \rho_{i, n} \frac{\int_{B_{r}(x)} V_{i} e^{u_{i, n}} d v_{g}}{\int_{\Sigma} V_{i} e^{u_{i, n}} d v_{g}} .
$$

Then,

$$
\left(\sigma_{1}(x), \sigma_{2}(x)\right) \in\{(0,0),(0,4 \pi),(4 \pi, 0),(4 \pi, 8 \pi),(8 \pi, 4 \pi),(8 \pi, 8 \pi)\} .
$$

In the same paper, the authors state that Theorem 1.1 immediately implies the following compactness result.

Theorem 1.2. Suppose $\alpha_{i j}=0$ for any $i, j$ and let $K_{1}, K_{2}$ be compact subsets of $\mathbb{R}^{+} \backslash 4 \pi \mathbb{N}$. Then, the space of solutions of (1) with $\rho_{i} \in K_{i}$ satisfying $\int_{\Sigma} u_{i} d v_{g}=0$ is compact in $H^{1}(\Sigma)$.

Theorem 1.2 is a necessary step to find solutions of (1) by variational methods, as was done in [2, 16, 17].

Although Theorem 1.2 has been widely used, it was not explicitly proved how it follows from Theorem [1.1. Recently, in [13], a proof was given in the case $\rho_{1}<8 \pi$.

The purpose of this note is to give a complete proof of Theorem 1.2 , extending it to the singular case as well. Actually, the proof follows quite directly from [7].

In the presence of singularities, that is when we allow the $\alpha_{i j}$ to be non-zero, it is convenient to write the system (11) in an equivalent form through the following change of variables:

$$
u_{i} \rightarrow u_{i}+4 \pi \sum_{j=1}^{l} \alpha_{i j} G_{p_{j}} \quad \text { where } G_{p} \text { solves }\left\{\begin{array}{l}
-\Delta G_{p}=\delta_{p}-\frac{1}{|\Sigma|} \\
\int_{\Sigma} G_{p} d v_{g}=0
\end{array} .\right.
$$

The new $u_{i}$ 's solve

$$
-\Delta u_{i}=\sum_{j=1}^{2} a_{i j} \rho_{j}\left(\frac{\widetilde{V}_{j} e^{u_{j}}}{\int_{\Sigma} \widetilde{V}_{j} e^{u_{j}} d v_{g}}-\frac{1}{|\Sigma|}\right) \quad i=1,2 .
$$

with

$$
\widetilde{V}_{i}=\prod_{j=1}^{l} e^{-4 \pi \alpha_{i j} G_{p_{j}}} V_{i} \quad \Rightarrow \quad \widetilde{V}_{i} \sim d\left(\cdot, p_{j}\right)^{2 \alpha_{i j}} \quad \text { near } p_{j} .
$$


In this case, we still have an analogue of Theorem 1.1 for the newly defined $u_{i}$. The finiteness of the local blow-up values has been proved in [14.

We will also show how this quantization result implies compactness of solutions outside a closed, zero-measure set of $\mathbb{R}^{+2}$.

Theorem 1.3. There exist two discrete subset $\Lambda_{1}, \Lambda_{2} \subset \mathbb{R}^{+}$, depending only on the $\alpha_{i j}$ 's, such that for any $K_{i} \Subset \mathbb{R}^{+} \backslash \Lambda_{i}$, the space of solutions of (11) with $\rho_{i} \in K_{i}$ satisfying $\int_{\Sigma} u_{i} d v_{g}=0$ is compact in $H^{1}(\Sigma)$.

As in the regular case, Theorem 1.3 has an important application in the variational analysis of (11), see for instance [2, 1].

\section{Proof of the main results}

Let us consider a sequence $u_{n}$ of solutions of (11) with $\rho_{i}=\rho_{i, n} \underset{n \rightarrow+\infty}{\rightarrow} \bar{\rho}_{i}$ and let us define

$$
w_{i, n}:=u_{i, n}-\log \int_{\Sigma} \widetilde{V}_{i} e^{u_{i, n}} d v_{g}+\log \rho_{i, n}
$$

which solves

$$
\left\{\begin{array}{l}
-\Delta w_{i, n}=\sum_{j=1}^{2} a_{i j}\left(\widetilde{V}_{j} e^{w_{j, n}}-\frac{\rho_{j, n}}{|\Sigma|}\right) \\
\int_{\Sigma} \widetilde{V}_{i} e^{w_{i, n}} d v_{g}=\rho_{i, n}
\end{array}\right.
$$

moreover,

$$
\sigma_{i}(x)=\lim _{r \rightarrow 0} \lim _{n \rightarrow+\infty} \int_{B_{r}(x)} \widetilde{V}_{i} e^{w_{i, n}} d v_{g}
$$

Let us denote by $S_{i}$ the blow-up set of $w_{i, n}$ :

$$
S_{i}:=\left\{x \in \Sigma: \exists\left\{x_{n}\right\} \subset \Sigma, w_{i, n}\left(x_{n}\right) \underset{n \rightarrow+\infty}{\longrightarrow}+\infty\right\}
$$

For $w_{i, n}$ we have a concentration-compactness result from [15, 3]:

Theorem 2.1. Up to subsequences, one of the following alternatives holds:

- (Compactness) $w_{i, n}$ is bounded in $L^{\infty}(\Sigma)$ for $i=1,2$. 
- (Blow-up) The blow-up set $S:=S_{1} \cup S_{2}$ is non-empty and finite and $\forall i \in\{1,2\}$ either $w_{i, n}$ is bounded in $L_{l o c}^{\infty}(\Sigma \backslash S)$ or $w_{i, n} \longrightarrow-\infty$ locally uniformly in $\Sigma \backslash S$.

In addition, if $S_{i} \backslash\left(S_{1} \cap S_{2}\right) \neq \emptyset$, then $w_{i, n} \longrightarrow-\infty$ locally uniformly in $\Sigma \backslash S$.

Moreover, denoting by $\mu_{i}$ the weak limit of the sequence of measures $\widetilde{V}_{i} e^{w_{i, n}}$, one has

$$
\mu_{i}=r_{i}+\sum_{x \in S_{i}} \sigma_{i}(x) \delta_{x}
$$

with $r_{i} \in L^{1}(\Sigma) \cap L_{l o c}^{\infty}\left(\Sigma \backslash S_{i}\right)$ and $\sigma_{i}(x) \geq 2 \pi \min \left\{1,1+\alpha_{i}(x)\right\} \forall x \in S_{i}$, $i=1,2$, where

$$
\alpha_{i}(x)= \begin{cases}0 & \text { if } x \neq p_{j} j=1, \ldots, l \\ \alpha_{i j} & \text { if } x=p_{j} .\end{cases}
$$

Here we want to show that one has $r_{i} \equiv 0$ for at least one $i \in\{1,2\}$. It may actually occur that only one of the $r_{i}$ 's is zero, as shown in [9]. Anyway, to prove Theorems 1.2 and 1.3 we only need one between $r_{1}$ and $r_{2}$ to be identically zero.

As a first thing, we can show that the profile near blow-up points resembles a combination of Green's functions:

Lemma 2.1. $w_{i, n}-\bar{w}_{i, n} \longrightarrow \sum_{j=1}^{2} \sum_{x \in S_{j}} a_{i j} \sigma_{j}(x) G_{x}+s_{i}$ in $L_{l o c}^{\infty}(\Sigma \backslash S)$ and weakly in $W^{1, q}(\Sigma)$ for any $q \in(1,2)$ with $e^{s_{i}} \in L^{p}(\Sigma) \forall p \geq 1$.

Proof. If $q \in(1,2)$

$$
\int_{\Sigma} \nabla w_{i, n} \cdot \nabla \varphi d v_{g} \leq\left\|\Delta w_{i, n}\right\|_{L^{1}(\Sigma)}\|\varphi\|_{\infty} \leq C\|\varphi\|_{W^{1, q^{\prime}}(\Sigma)}
$$

$\forall \varphi \in W^{1, q^{\prime}}(\Sigma)$ with $\int_{\Sigma} \varphi=0$, hence one has $\left\|\nabla w_{i, n}\right\|_{L^{q}(\Sigma)} \leq C$. In particular $w_{i, n}-\bar{w}_{i, n}$ converges to a function $w_{i} \in W^{1, q}(\Sigma)$ weakly in $W^{1, q}(\Sigma) \forall q \in(1,2)$ and, thanks to standard elliptic estimates, we get convergence in $L_{l o c}^{\infty}(\Sigma \backslash S)$.

The limit functions $w_{i}$ are distributional solutions of

$$
-\Delta w_{i}=\sum_{j=1}^{2} a_{i j}\left(r_{j}+\sum_{x \in S_{j}} \sigma_{j}(x) \delta_{x}-\frac{\bar{\rho}_{j}}{|\Sigma|}\right) .
$$


In particular $s_{i}:=w_{i}-\sum_{j=1}^{2} \sum_{x \in S_{j}} a_{i j} \sigma_{j}(x) G_{x}$ solves

$$
-\Delta s_{i}=\sum_{j=1}^{2} a_{i j}\left(r_{j}+\frac{1}{|\Sigma|} \sum_{x \in S_{j}} \sigma_{j}(x)-\frac{\bar{\rho}_{j}}{|\Sigma|}\right) .
$$

Since $-\Delta s_{i} \in L^{1}(\Sigma)$ we can exploit Remark 2 in [5] to prove that $e^{s_{i}} \in L^{p}(\Sigma) \forall p \geq 1$.

The following Lemma shows the main difference between the case of vanishing and non-vanishing residual.

\section{Lemma 2.2.}

- $r_{i} \equiv 0 \Longrightarrow \bar{w}_{i, n} \longrightarrow-\infty$.

- $r_{i} \not \equiv 0 \Longrightarrow \bar{w}_{i, n}$ is bounded.

Proof. First of all, $\bar{w}_{i, n}$ is bounded from above due to Jensen's inequality.

Now, take any non-empty open set $\Omega \Subset \Sigma \backslash S$.

$$
\int_{\Omega} \widetilde{V}_{i} e^{w_{i, n}} d v_{g}=e^{\bar{w}_{i, n}} \int_{\Omega} \widetilde{V}_{i} e^{w_{i, n}-\bar{w}_{i, n}} d v_{g}
$$

and by Lemma 2.1

$$
\int_{\Omega} \widetilde{V}_{i} e^{w_{i, n}-\bar{w}_{i, n}} d v_{g} \underset{n \rightarrow+\infty}{\longrightarrow} \int_{\Omega} \widetilde{V}_{i} e^{\sum_{j=1}^{2} \sum_{x \in S_{j}} a_{i j} \sigma_{j}(x) G_{x}+s_{i}} d v_{g} \in(0,+\infty) .
$$

On the other hand,

$$
\int_{\Omega} \widetilde{V}_{i} e^{w_{i, n}} d v_{g} \underset{n \rightarrow+\infty}{\longrightarrow} \mu_{i}(\Omega)=\int_{\Omega} r_{i}(x) d v_{g}(x) .
$$

If $r_{i} \equiv 0$ one has $\bar{w}_{i, n} \longrightarrow-\infty$. If instead $r_{i} \not \equiv 0$, choosing $\Omega$ such that $\int_{\Omega} r_{i}(x) d v_{g}>0$ we must have $\bar{w}_{i, n}$ necessarily bounded.

Remark 2.1. From the previous two lemmas, we can write $r_{i}=\widehat{V}_{i} e^{s_{i}}$, where

$$
\widehat{V}_{i}:=\widetilde{V}_{i} e^{\lim _{n \rightarrow+\infty} \bar{w}_{i, n}} e^{\sum_{j=1}^{2} \sum_{x \in S_{j}} a_{i j} \sigma_{j}(x) G_{x}}
$$

satisfies $\widehat{V}_{i} \sim d(\cdot, x)^{2 \alpha_{i}(x)-\frac{\sum_{j=1}^{2} a_{i j} \sigma_{j}(x)}{2 \pi}}$ around each $x \in S_{i}$, provided $r_{i} \not \equiv 0$. 
The key Lemma is an extension of Chae-Ohtsuka-Suzuki [7 to the singular case. Basically, it gives necessary conditions on the $\sigma_{i}$ 's to have non-vanishing residual.

Lemma 2.3. For both $i=1,2$ we have $s_{i} \in W^{2, p}(\Sigma)$ for some $p>1$. Moreover, if $\sum_{j=1}^{2} a_{i j} \sigma_{j}\left(x_{0}\right) \geq 4 \pi\left(1+\alpha_{i}\left(x_{0}\right)\right)$ for some $x_{0} \in S_{i}$, then $r_{i} \equiv 0$.

Proof. If both $r_{1}$ and $r_{2}$ are identically zero, then also $s_{1}$ and $s_{2}$ are both identically zero, so there is nothing to prove.

Suppose now $r_{1} \not \equiv 0$ and $r_{2} \equiv 0$. In this case,

$$
\left\{\begin{array}{l}
-\Delta s_{1}=2\left(r_{1}+\frac{1}{|\Sigma|} \sum_{x_{0} \in S_{1}} \sigma_{1}\left(x_{0}\right)-\frac{\bar{\rho}_{1}}{|\Sigma|}\right) \\
-\Delta s_{2}=-\left(r_{1}+\frac{1}{|\Sigma|} \sum_{x_{0} \in S_{1}} \sigma_{1}\left(x_{0}\right)-\frac{\bar{\rho}_{1}}{|\Sigma|}\right)
\end{array} .\right.
$$

Then, being $G_{x}(y) \geq-C$ for all $x, y \in \Sigma$ with $x \neq y$, we get

$$
s_{1}(x)=\int_{\Sigma} G_{x}(y) 2 r_{1}(y) d v_{g}(y) \geq-2 C \int_{\Sigma} r_{1} d v_{g} \geq-C^{\prime} .
$$

Therefore, from the previous remark, around each $x_{0} \in S_{1}$ we get

$$
r_{1}(y) \geq C d\left(x_{0}, y\right)^{2 \alpha_{1}\left(x_{0}\right)-\frac{\sum_{j=1}^{2} a_{1 j} \sigma_{j}\left(x_{0}\right)}{2 \pi}},
$$

so being $r_{1} \in L^{1}(\Sigma)$, it must be $\sum_{j=1}^{2} a_{1 j} \sigma_{j}\left(x_{0}\right)<4 \pi\left(1+\alpha_{1}\left(x_{0}\right)\right)$.

Moreover, being $e^{q s_{1}} \in L^{1}(\Sigma)$ for any $q \geq 1$, from Holder's inequality we get $r_{1} \in L^{p}(\Sigma)$ for some $p>1$; therefore, standard estimates yield $s_{i} \in W^{2, p}(\Sigma)$ for both $i=1,2$.

Consider now the case of both non-vanishing residuals, which means by Theorem $2.1 S_{1}=S_{2}=S$. In this case,

$$
-\Delta\left(\frac{2 s_{1}+s_{2}}{3}\right)=\left(r_{1}+\frac{1}{|\Sigma|} \sum_{x_{0} \in S_{1}} \sigma_{1}\left(x_{0}\right)-\frac{\bar{\rho}_{1}}{|\Sigma|}\right)
$$

hence, arguing as before, $\frac{2 s_{1}+s_{2}}{3} \geq-C$. Therefore, using the convexity of $t \rightarrow e^{t}$ we get

$$
\begin{gathered}
C \int_{\Sigma} \min \left\{\widehat{V}_{1}, \widehat{V}_{2}\right\} d v_{g} \leq \int_{\Sigma} \min \left\{\widehat{V}_{1}, \widehat{V}_{2}\right\} e^{\frac{2 s_{1}+s_{2}}{3}} d v_{g} \leq \\
\leq \frac{2}{3} \int_{\Sigma} \widehat{V}_{1} e^{s_{1}} d v_{g}+\frac{1}{3} \int_{\Sigma} \widehat{V}_{2} e^{s_{2}} d v_{g}=\frac{2}{3} \int_{\Sigma} r_{1} d v_{g}+\frac{1}{3} \int_{\Sigma} r_{2} d v_{g}<+\infty .
\end{gathered}
$$


Therefore, for any $x_{0} \in S$ there exists $i \in\{1,2\}$ such that $\sum_{j=1}^{2} a_{i j} \sigma_{j}\left(x_{0}\right)<$ $4 \pi\left(1+\alpha_{i}\left(x_{0}\right)\right)$. Fix $x_{0}$ and suppose, without loss of generality, that this is true for $i=1$. This implies that $r_{1} \in L^{p}\left(B_{r}\left(x_{0}\right)\right)$ for small $r$, so for $x \in B_{\frac{r}{2}}\left(x_{0}\right)$ we have

$$
\begin{aligned}
s_{2}(x) & =\int_{\Sigma} G_{x}(y) 2 r_{2}(y) d v_{g}(y)-\int_{B_{r}\left(x_{0}\right)} G_{x}(y) r_{1}(y) d v_{g}(y) \\
& -\int_{\Sigma \backslash B_{r}\left(x_{0}\right)} G_{x}(y) r_{1}(y) d v_{g}(y) \\
& \geq-C-\sup _{z \in \Sigma}\left\|G_{z}\right\|_{L^{p^{\prime}(\Sigma)}}\left\|r_{1}\right\|_{L^{p}\left(B_{r}\left(x_{0}\right)\right)} \\
& -\sup _{z \in B_{\frac{r}{2}}\left(x_{0}\right)}\left\|G_{z}\right\|_{L^{\infty}\left(\Sigma \backslash B_{r}\left(x_{0}\right)\right)}\left\|r_{1}\right\|_{L^{1}(\Sigma)} \\
& \geq-C^{\prime} .
\end{aligned}
$$

Therefore, arguing as before, we must have $\sum_{j=1}^{2} a_{2 j} \sigma_{j}\left(x_{0}\right)<4 \pi(1+$ $\left.\alpha_{2}\left(x_{0}\right)\right)$ and $r_{2} \in L^{p}\left(B_{\frac{r}{2}}\left(x_{0}\right)\right)$. This implies $-\Delta s_{i} \in L^{p}\left(B_{\frac{r}{2}}\left(x_{0}\right)\right)$ for both $i$ 's. Hence, being $x_{0}$ arbitrary and $-\Delta s_{i} \in L_{l o c}^{p}(\Sigma \backslash S)$, by elliptic estimates the proof is complete.

From Lemmas 2.1 and 2.3 we can deduce, through a Pohozaev identity, the following information about the local blow-up values. This was explicitly done in [12, 14].

Lemma 2.4. If $x_{0} \in S$ then

$\sigma_{1}^{2}\left(x_{0}\right)+\sigma_{2}^{2}\left(x_{0}\right)-\sigma_{1}\left(x_{0}\right) \sigma_{2}\left(x_{0}\right)=4 \pi\left(1+\alpha_{1}\left(x_{0}\right)\right) \sigma_{1}\left(x_{0}\right)+4 \pi\left(1+\alpha_{2}\left(x_{0}\right)\right) \sigma_{2}\left(x_{0}\right)$.

Lemma 2.5. If $x_{0} \in S_{1} \cap S_{2}$ then there exists $i$ such that $\sum_{j=1}^{2} a_{i j} \sigma_{j}\left(x_{0}\right) \geq$ $4 \pi\left(1+\alpha_{i}\left(x_{0}\right)\right)$.

Proof. Suppose the statement is not true. Then, by Lemmas 2.3 and 2.4, we would have

$$
\left\{\begin{array}{l}
2 \sigma_{1}\left(x_{0}\right)-\sigma_{2}\left(x_{0}\right)<4 \pi\left(1+\alpha_{1}\left(x_{0}\right)\right) \\
2 \sigma_{2}\left(x_{0}\right)-\sigma_{1}\left(x_{0}\right)<4 \pi\left(1+\alpha_{2}\left(x_{0}\right)\right) \\
\sigma_{1}^{2}\left(x_{0}\right)+\sigma_{2}^{2}\left(x_{0}\right)-\sigma_{1}\left(x_{0}\right) \sigma_{2}\left(x_{0}\right)= \\
=4 \pi\left(1+\alpha_{1}\left(x_{0}\right)\right) \sigma_{1}\left(x_{0}\right)+4 \pi\left(1+\alpha_{2}\left(x_{0}\right)\right) \sigma_{2}\left(x_{0}\right)
\end{array}\right.
$$

which has no solution between positive $\sigma_{1}\left(x_{0}\right), \sigma_{2}\left(x_{0}\right)$.

In fact, by multiplying the first equation by $\frac{\sigma_{1}\left(x_{0}\right)}{2}$ and the second by 
$\frac{\sigma_{2}\left(x_{0}\right)}{2}$ and summing, we get

$\sigma_{1}^{2}\left(x_{0}\right)+\sigma_{2}^{2}\left(x_{0}\right)-\sigma_{1}\left(x_{0}\right) \sigma_{2}\left(x_{0}\right)<2 \pi\left(1+\alpha_{1}\left(x_{0}\right)\right) \sigma_{1}\left(x_{0}\right)+2 \pi\left(1+\alpha_{2}\left(x_{0}\right)\right) \sigma_{2}\left(x_{0}\right)$,

which contradicts the third equation.

The scenario is described by the picture.

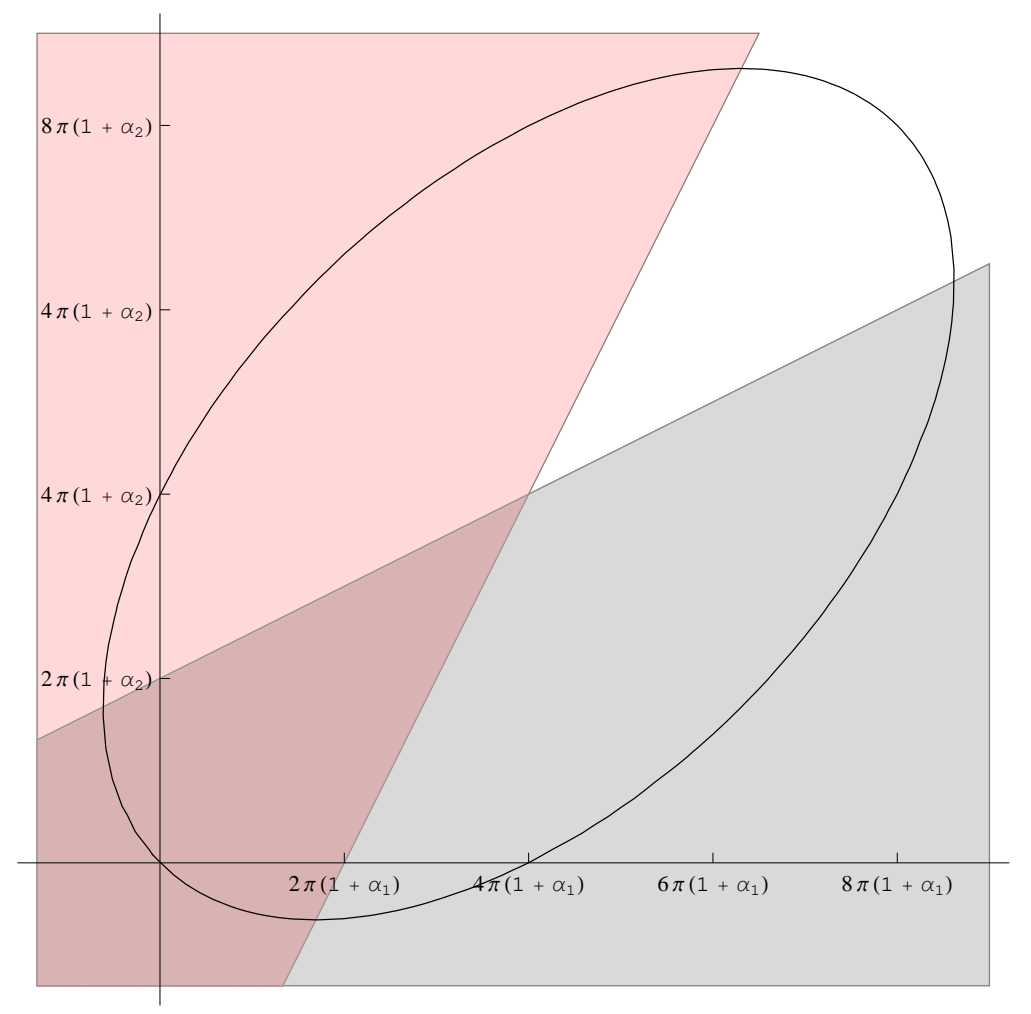

Figure 1: The algebraic conditions (7) satisfied by $\sigma_{1}\left(x_{0}\right), \sigma_{2}\left(x_{0}\right)$

Corollary 2.1. Let $w_{n}$ be a sequence of solutions of (6) . If $S \neq \emptyset$ then either $r_{1} \equiv 0$ or $r_{2} \equiv 0$. In particular there exists $i \in\{1,2\}$ such that $\bar{\rho}_{i}=\sum_{x \in S_{i}} \sigma_{i}(x)$.

\section{Proof of Theorems 1.2 and 1.3 .}

Let $u_{n}$ be a sequence of solutions of (11) with $\rho_{i}=\rho_{i, n} \underset{n \rightarrow+\infty}{\longrightarrow} \bar{\rho}_{i}$ and $\int_{\Sigma} u_{1, n} d v_{g}=\int_{\Sigma} u_{2, n} d v_{g}=0$ and let $w_{i, n}$ be defined by (5).

If both $w_{1, n}$ and $w_{2, n}$ are bounded from above, then by standard estimates $u_{n}$ is bounded in $W^{2, p}(\Sigma)$, hence is compact in $H^{1}(\Sigma)$. 
Otherwise, from Corollary 2.1 we must have $\bar{\rho}_{i}=\sum_{x \in S_{i}} \sigma_{i}(x)$ for some $i \in\{1,2\}$. In the regular case, from Theorem 1.1 follows that $\rho_{i}$ must be an integer multiple of $4 \pi$, hence the proof of Theorem 1.2 is complete.

In the singular case, local blow-up values at regular points are still defined by (3), whereas for any $j=1, \ldots, l$ there exists a finite $\Gamma_{j}$ such that $\left(\sigma_{1}\left(p_{j}\right), \sigma_{2}\left(p_{j}\right)\right) \in \Gamma_{j}$. Therefore, it must hold

$$
\rho_{i} \in \Lambda_{i}:=\left\{4 \pi k+\sum_{j=1}^{l} n_{j} \sigma_{j}, k \in \mathbb{N}, n_{j} \in\{0,1\}, \sigma_{j} \in \Pi_{i}\left(\Gamma_{j}\right)\right\},
$$

where $\Pi_{i}$ is the projection on the $i^{\text {th }}$ component; being $\Lambda_{i}$ discrete we can also conclude the proof of Theorem 1.3 .

\section{Acknowledgments}

The authors have been supported by the PRIN project Variational and perturbative aspects of nonlinear differential problems.

\section{References}

[1] L. Battaglia. Existence and multiplicity result for the singular Toda system. J. Math. Anal. Appl., 424(1):49-85, 2015.

[2] L. Battaglia, A. Jevnikar, A. Malchiodi, and D. Ruiz. A general existence result for the Toda System on compact surfaces. arXiv 1306.5404, 2013.

[3] L. Battaglia and A. Malchiodi. A Moser-Trudinger inequality for the singular Toda system. Bull. Inst. Math. Acad. Sin. (N.S.), $9(1): 1-23,2014$.

[4] J. Bolton and L. M. Woodward. Some geometrical aspects of the 2-dimensional Toda equations. In Geometry, topology and physics (Campinas, 1996), pages 69-81. de Gruyter, Berlin, 1997.

[5] H. Brezis and F. Merle. Uniform estimates and blow-up behavior for solutions of $-\Delta u=V(x) e^{u}$ in two dimensions. Comm. Partial Differential Equations, 16(8-9):1223-1253, 1991. 
[6] E. Calabi. Isometric imbedding of complex manifolds. Ann. of Math. (2), 58:1-23, 1953.

[7] D. Chae, H. Ohtsuka, and T. Suzuki. Some existence results for solutions to SU(3) Toda system. Calc. Var. Partial Differential Equations, 24(4):403-429, 2005.

[8] S. S. Chern and J. G. Wolfson. Harmonic maps of the twosphere into a complex Grassmann manifold. II. Ann. of Math. (2), 125(2):301-335, 1987.

[9] T. D'Aprile, A. Pistoia, and D. Ruiz. Asymmetric blow-up for the $S U(3)$ Toda System. arXiv 1411.3482, 2014.

[10] G. Dunne. Self-dual Chern-Simons Theories. Lecture notes in physics. New series m: Monographs. Springer, 1995.

[11] J. Jost, C. Lin, and G. Wang. Analytic aspects of the Toda system. II. Bubbling behavior and existence of solutions. Comm. Pure Appl. Math., 59(4):526-558, 2006.

[12] J. Jost and G. Wang. Analytic aspects of the Toda system. I. A Moser-Trudinger inequality. Comm. Pure Appl. Math., 54(11):1289-1319, 2001.

[13] C. Lin, J. Wei, and W. Yang. Degree counting and shadow system for $S U(3)$ Toda system: one bubbling. arXiv 1408.5802, 2014.

[14] C. Lin, J. Wei, and L. Zhang. Classification of blowup limits for SU(3) singular Toda systems. arXiv 1303.4167, 2014.

[15] M. Lucia and M. Nolasco. SU(3) Chern-Simons vortex theory and Toda systems. J. Differential Equations, 184(2):443-474, 2002.

[16] A. Malchiodi and C. B. Ndiaye. Some existence results for the Toda system on closed surfaces. Atti Accad. Naz. Lincei Cl. Sci. Fis. Mat. Natur. Rend. Lincei (9) Mat. Appl., 18(4):391-412, 2007.

[17] A. Malchiodi and D. Ruiz. A variational analysis of the Toda system on compact surfaces. Comm. Pure Appl. Math., 66(3):332371, 2013. 
[18] G. Tarantello. Selfdual gauge field vortices. Progress in Nonlinear Differential Equations and their Applications, 72. Birkhäuser Boston Inc., Boston, MA, 2008. An analytical approach.

[19] Y. Yang. Solitons in field theory and nonlinear analysis. Springer Monographs in Mathematics. Springer-Verlag, New York, 2001. 